\title{
Design and Fabrication of 77GHz HEMT Mixer Modules using Experimentally Optimized Antipodal Finline Transitions
}

\author{
Dong-Wook Kim, Seung-Won Paek, Jae-Hak Lee, Kye-Ik Jeon, \\ Chae-Rok Lim, Young-Woo Kwon*, Ki-Woong Chung \\ RF Device Team, LG Corporate Institute of Technology(LG-CIT) \\ 16 Woomyeon-Dong, Seocho-Gu, Seoul 137-724, Korea \\ * School of Electrical Engineering, Seoul National University \\ San 56-1 Shilim-Dong, Kwanak-Gu, Seoul 151-742, Korea
}

\begin{abstract}
GHz PHEMT gate mixer module and resistive mixer module were fabricated for automotive applications using LG-CIT low noise PHEMT process and WR12-to-microstrip antipodal finline transitions. Experimental optimization of the finline transitions was attempted for wideband operation and low insertion loss by adjusting geometrical design parameters. The average insertion loss was measured to be $0.74 \mathrm{~dB}$ per transition in $75 \sim 90 \mathrm{GHz}$. The fabricated gate mixer module and the resistive mixer module showed very good conversion loss of $2 \mathrm{~dB}$ and $10.3 \mathrm{~dB}$, respectively, including finline transition loss and IF cable loss, which were very competitive performances.
\end{abstract}

\section{INTRODUCTION}

Car radars and sensors have been one of the most important and interesting areas in the microwave and millimeter wave applications over the past years [1]. These not only are related to huge market of automotive industry but also play a key role in safety of intelligent cruise control in the future. Among the components of the radars and sensors operating at $77 \mathrm{GHz}$ for short range and high resolution applications, mixer is one of the most important circuit elements that converts Doppler-shifted reflected signal to very low frequency signal below $1 \mathrm{MHz}$.

For the fabrication of millimeter wave mixer modules, coaxial-to-microstrip transition does not have good reproducibility and requires fine and accurate mechanical machining which leads to higher cost. To overcome these difficulties, direct waveguide-to-microstrip transition techniques have been widely used [2-3]. We used antipodal finline transition so as to achieve wide bandwidth and assembly easiness in our works.

In this paper, we present the measured results of experimentally optimized WR-12 to microstrip transitions. And also we present the design, fabrication and measured results of $77 \mathrm{GHz}$ HEMT gate mixer module and resistive mixer module that were mounted on WR-12 waveguide jigs using these optimized finline transitions.

\section{WAVEGUIDE-TO-MICROSTRIP TRANSITION}

The insertion of the antipodal finline substrate into rectangular waveguide makes the dominant $\mathrm{TE}_{10}$ mode of 
the waveguide rotate $90^{\circ}$ and form the electric field of the microstrip line, which is illustrated in Fig. 1. This finline transition is not sensitive to mechanical machining and mechanical parameter variation. But the characteristics of the antipodal finline transition could not be accurately predicted only by three-dimensional electromagnetic field (EM) simulation. We optimized the structure of the transition by simultaneously performing FDTD (Finite Difference Time Domain) simulation and experimental optimization of the structure design parameters. The major design parameters are as follows: the transfer length for low insertion loss (tp), the shape and length of ground semicircular pattern for in-band resonance removal $(\mathrm{S}, \mathrm{L})$, the alignment of top and bottom (signal and ground) patterns and so on, as shown in Fig. 1. The serrated choke $\left(\mathrm{W}_{1}, \mathrm{~W}_{2}, \mathrm{~W}_{3}\right)$ was also used for fragility prevention and reproducibility during the assembly. Impedance discontinuity between the waveguide and the microstrip was reduced using low dielectric constant quartz substrate whose $\varepsilon_{\mathrm{r}}$ is 2.2 .

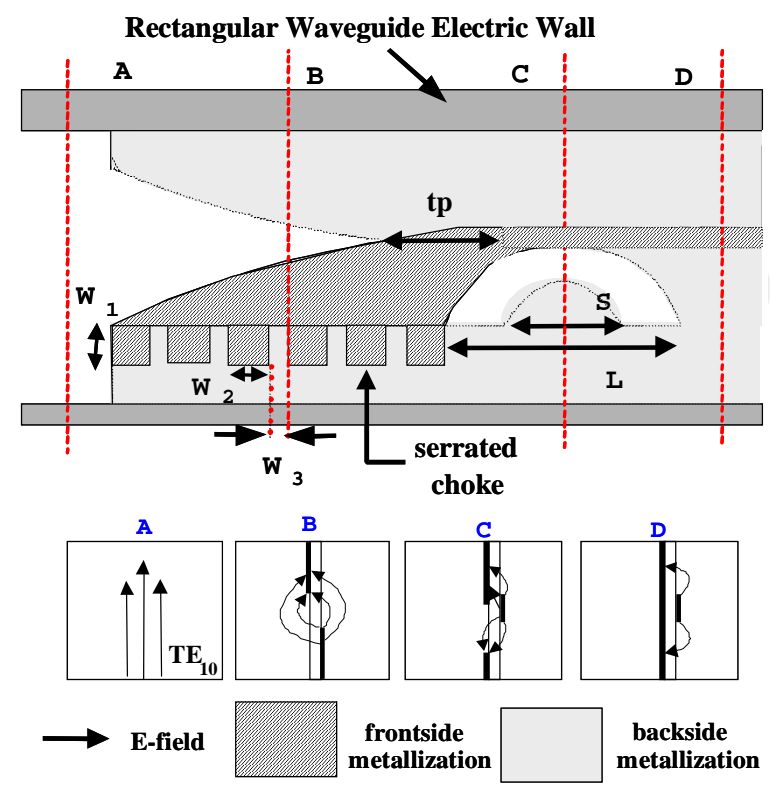

Fig. 1 THE STRUCTURE AND DESIGN PARAMETERS OF THE ANTIPODAL FINLINE TRANSITION (A, B, C AND D LINES SHOW THE FIELD ROTATION AS THE WAVE PROPAGATES TOWARD INSERTED TRANSITION.)
We found from our experiments that long transfer length showed relatively lower insertion loss than short one, semicircular pattern caused no remarkable half-wave resonance predicted from EM simulations but reduced the ripples of insertion loss, and the serrated choke worked well for RF grounding in the wide frequency range.

The optimized transition showed average insertion loss of $0.74 \mathrm{~dB}$ per transition in the frequency range of 75 90GHz. Fig. 2 shows measured results of back-toback WR-12 to microstrip antipodal finline transitions.

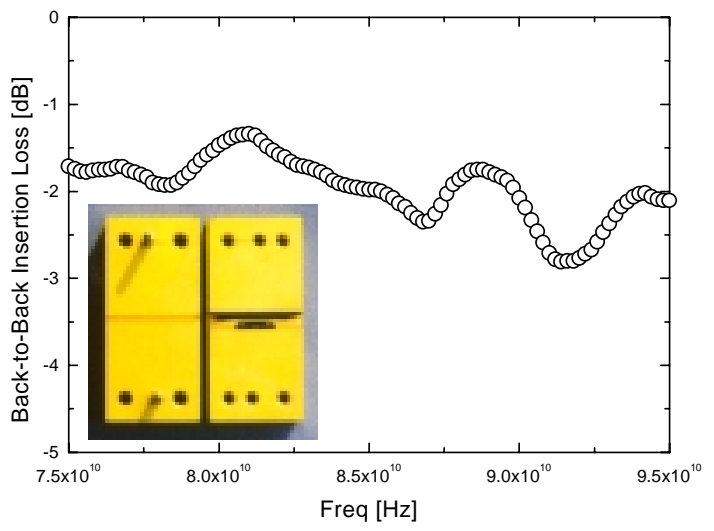

Fig. 2 THE MEASURED INSERTION LOSS OF BACK-TOBACK ANTIPODAL FINLINE TRANSITIONS (THE AVERAGE INSERTION LOSS OF 0.74DB WAS OBTAINED PER TRANSITION IN 75GHZ 90GHZ.)

\section{PHEMT GATE MIXER MODULE}

We designed and fabricated hybrid gate mixer module in which the mixing operation was mainly caused by the nonlinearity of the transconductance in active device, PHEMT. The matching circuits at RF and LO frequencies were fabricated on 5 mil quartz substrate with waveguide transitions. The photograph of the gate mixer module is shown in Fig. 3. The measured conversion loss is shown in Fig. 4. The results showed very good conversion loss of $2 \mathrm{~dB}$ with $\mathrm{RF}$ power of $-25.45 \mathrm{dBm}$ at $76.878 \mathrm{GHz}$ and 
LO power of $11.93 \mathrm{dBm}$ at $76.495 \mathrm{GHz}$. The conversion loss data included RF loss of the waveguide-to-microstrip antipodal finline transition and IF loss of bias tee and SMA cable.

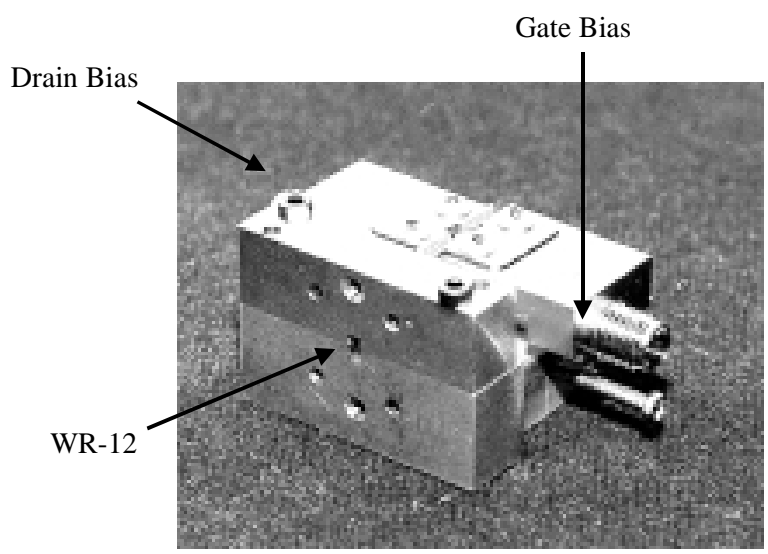

Fig. 3 THE FABRICATED GATE MIXER MODULE WITH OPTIMIZED FINLINE TRANSITIONS

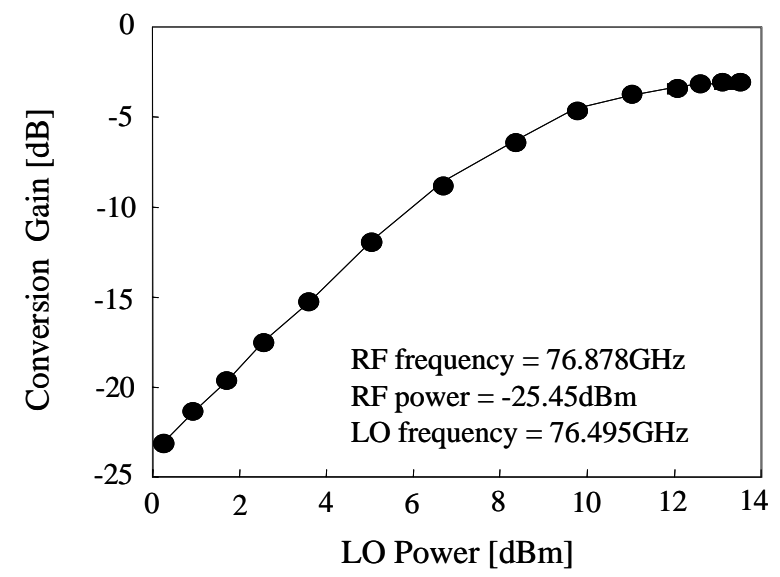

Fig. 4 THE MEASURED CONVERSION GAIN OF THE GATE MIXER MODULE

\section{PHEMT RESISTIVE MMIC MIXER MODULE}

We also fabricated $77 \mathrm{GHz}$ HEMT resistive MMIC mixer on 3" GaAs semi-insulating substrate that had single hetero-epitaxial P-HEMT layers grown by MBE (Molecular Beam Epitaxy). We utilized double exposuredouble develop technique [4] to improve uniformity and reproducibility of $\mathrm{T}$-gate process that was a requisite for high speed and high frequency operation. The resistive mixer was designed using the Root model [5] of LG-CIT low noise P-HEMT. The photograph of the fabricated MMIC mixer is presented in Fig. 5. The MMIC chip size is $1.2 \mathrm{~mm} \times 1.0 \mathrm{~mm}$.

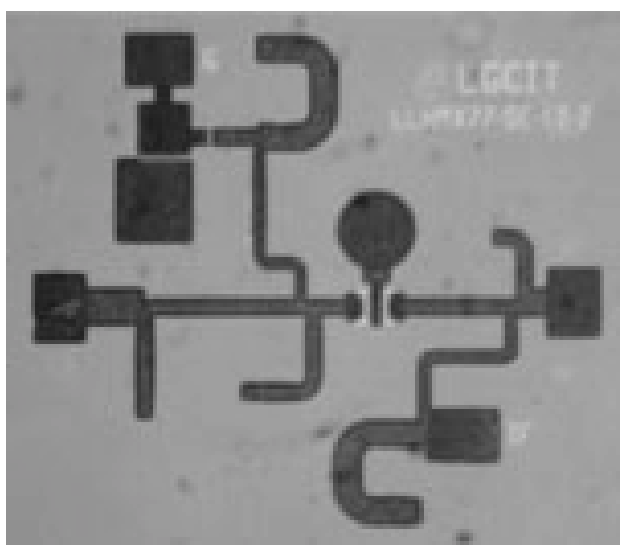

Fig. 5 THE PHOTOGRAPH OF THE FABRICATED P-HEMT RESISTIVE MMIC MIXER (SIZE: $1.2 \mathrm{~mm} \times 1.0 \mathrm{~mm})$

The MMIC chip was mounted on the waveguide module jig, which included WR-12 to microstrip finline transitions. The fabricated mixer module is shown along with detailed MMIC die attach photograph in Fig. 6. The MMIC mixer chip is indicated by small box and arrow line. The relatively large bias SMA port and IF SMA port were inserted for test convenience. The measurements were done using Gunn oscillator as signal source and WR-12 waveguide accessories such as variable attenuator, directional coupler, harmonic mixer and so on. The measured conversion loss of the mixer module with LO frequency of $76.6955 \mathrm{GHz}$ and RF power of $-15 \mathrm{dBm}$ at $76.6690 \mathrm{GHz}$ is shown in Fig. 7. As shown in Fig. 7, the conversion loss of $10.3 \mathrm{~dB}$ was obtained at $\mathrm{LO}$ power of $7 \mathrm{dBm}$ that was very competitive performance. This is in good agreement with simulation result. 


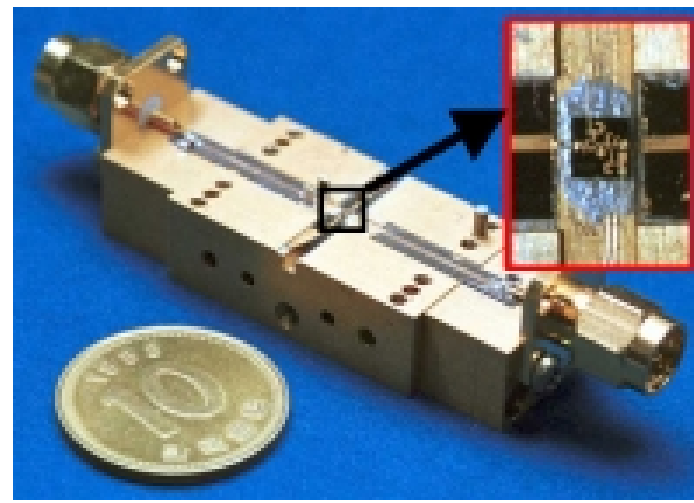

Fig. 6 THE FABRICATED 77GHZ RESISTIVE MIXER MODULE THAT UTILIZES WR-12 WAVEGUIDE AND WR12 TO MICROSTRIP FINLINE TRANSITIONS

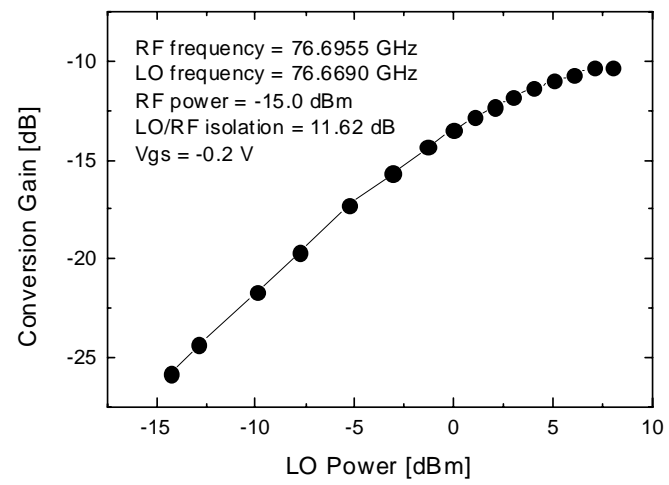

Fig. 7 THE MEASURED CONVERSION GAIN OF THE 77GHZ MIXER MODULE WITH LO POWER AT 76.6955GHZ AND RF POWER AT 76.6690GHZ

\section{CONCLUSIONS}

The optimized waveguide-to-microstrip antipodal finline structure was fabricated on 5 mil quartz substrate for wideband module applications. The measurements resulted in the average insertion loss of $0.74 \mathrm{~dB}$ per transition in the frequency range of $75 \sim 90 \mathrm{GHz}$. Using these transitions, we designed, fabricated and measured $77 \mathrm{GHz}$ PHEMT gate mixer module and resistive mixer module. The measured results of the gate mixer module presented very good conversion loss of about $2 \mathrm{~dB}$ with
RF power of $-25.45 \mathrm{dBm}$ at $76.878 \mathrm{GHz}$ and $\mathrm{LO}$ power of $11.93 \mathrm{dBm}$ at $76.495 \mathrm{GHz}$. And the results of the resistive mixer module showed competitive conversion loss of $10.3 \mathrm{~dB}$ with $\mathrm{RF}$ power of $-15 \mathrm{dBm}$ at $76.6955 \mathrm{GHz}$ and LO power of $7 \mathrm{dBm}$ at $76.6690 \mathrm{GHz}$ including the loss of finline transitions. Both modules were successfully fabricated and tested using experimentally optimized WR-12 to microstrip antipodal finline transitions on $5 \mathrm{mil}$ quartz substrate.

\section{ACKNOWLEDGEMENTS}

This work was funded in part by LG Precision, Co., which worked with Hyundai Motor Company for G7 Project.

\section{REFERENCES}

[1] Hiroshige Fukuhara, "Millimeter-Wave Radar and Potential Automotive Applications", in MWE '93 Microwave Workshop, pp. 227-232.

[2] D. I. Stones, “Analysis of a Novel Microstrip-to-Waveguide Transition/Combiner", in IEEE MTT-S Int'l Microwave Smposium, pp. 217-220, 1994.

[3] L. J. Lavedan, “ Design of Waveguide-to-Microstrip Transitions Specially Suited to Millimeter-Wave Applications", Electron. Letters, Vol. 13, No. 20, pp. 604605, Sep. 1977.

[4] Seung-Won Paek and Kwang-Seok Seo, “Fabrication of $0.2 \mu \mathrm{m}$ T-gate Low Noise Pseudomorphic HEMT by using Double E-beam Exposure Technique", in $3^{\text {rd }}$ Korean Conference on Semiconductor, pp. 25-26, Feb. 1996

[5] David E. Root and Siqi Fan, "Experimental Evaluation of Large-Signal Modeling Assumptions Based on Vector Analysis of Bias-Dependent S-Parameter Data from MESFETs and HEMTs", in 1992 IEEE MTT-S Int'l Microwave Symposium, pp. 255-258. 